\title{
Macromolecular X-ray Crystallography in the Undergraduate Curriculum
}

\author{
K McLaughlin ${ }^{1}$ \\ ${ }^{1}$ Department of Chemistry, Vassar College, Poughkeepsie, NY USA \\ kmclaughlin@vassar.edu
}

Though macromolecular x-ray crystallography has long been the primary method for protein and nucleic acid structure solution, the technique is often first introduced at the graduate level. However, macromolecular structures are encountered frequently throughout the undergraduate biological sciences curriculum, but their meaning and utility are not fully realized by students. Here I will discuss strategies to integrate macromolecular x-ray crystallography at the undergraduate level. Activities to introduce students to protein x-ray crystallography utilizing freely available resources such as the RCSB Protein Data Bank and COOT will be described. Design and implementation of a semester long biochemistry lab CURE (Course-based Undergraduate Research Experience) using protein structure as the driving theme will also be discussed.

Acta Cryst. (2020). A76, a13 